\title{
Study of Polarization Evolution in Phantom Tissues with Ultrafast Optics Techniques: Monte Carlo Simulations and Experiments
}

\author{
Xueding Wang and Lihong V. Wang \\ Optical Imaging Laboratory, Biomedical Engineering Program, Texas A\&M University, TX 77843-3120, U.S.A. \\ URL: http://oilab.tamu.edu \\ Chia-Wei Sun, Hsiang-Shi Wang, C. C. Yang and Yean-Woei Kiang \\ Department of Electrical Engineering, Graduate Institute of Electro-Optical Engineering, and Graduate Institute of \\ Communication Engineering, National Taiwan University, 1, Roosevelt Road, Sec. 4, Taipei, Taiwan, R.O.C.
(phone) 886-2-23657624
(fax) 886-2-23652637
(E-mail) ccy@cc.ee.ntu.edu.tw

\begin{abstract}
This paper presents our study results of polarized short pulse transmission through phantom tissues made of polystyrene particle solutions with various concentrations and particle sizes. To improve the quality of optical imaging using an ultrafast light source, study is required to fully understand the evolution of the polarization state in the sample, as well as the time- and polarization-dependent distributions of optical intensity exiting from samples. Temporal profiles of the Stokes vectors and the degree of polarization are measured experimentally. The results agree well with those resulting from Monte Carlo simulations. Analyses based on the StokesMueller formalism show that the first seattering event determines the spatial patterns of the transmitted Stokes vectors. When a detected area at the output surface of the sample is symmetric about the incident beam, the temporal profile of transmittance is independent of the incident polarization state. The linear relationship between the average order of scatters and the light propagation time can be used to explain the exponential decay of the degree of polarization and the inversely proportional relationship between the FWHM of the degree of polarization and the scatterer concentration. Keywords - Polarize, scattering, time-resolved, Monte Carlo
\end{abstract}

\section{INTRODUCTION}

Ultrafast optics has become a commonly accepted approach to enhance the optical imaging and diagnosis. With this technique, a short light pulse is applied to the biological tissues, and then the transmitted ballistic and snake photons that carry more information of tissue properties are extracted through various gating techniques [1-3]. In order to improve the quality of optical imaging using an ultrafast light source, more study is needed to fully understand the evolution of polarization in turbid media. Monte Carlo (MC) simulation offers a flexible and accurate approach to this problem $[4,5]$ because it can deal with complex geometries in both forward and backward manners, and can score multiple physical quantities simultaneously. We use a time-resolved MC technique to analyze the propagation of polarized light in randomly scattering turbid media. The accuracy of this $\mathrm{MC}$ algorithm has been proved by the results measured in experiments.

\section{METHODS}

The experimental setup is shown in Fig. 1(a). A Ti:Sapphire laser is used to provide $800 \mathrm{~nm}$ light pulses with a FWHM at $100 \mathrm{fs}$. The laser beam is split into two branches, one for triggering the streak camera and the other for propagating through the sample. The transmitted light from the sample is directed to the streak camera with a fiber bundle that has a receiving area of $1 \mathrm{~mm}$ diameter and a receiving angle of $30^{\circ}$. The temporal resolution of the operation mode of the streak camera is $4.74 \mathrm{ps}$. With the expansion of the optical signal in the fiber bundle and the limited resolution of the streak camera, the impulse response of the detection system has a FWHM of 20 ps. Tissue phantom is made of polystyrene microspheres solution ( $0.133 \%$ concentration and $380 \mathrm{~nm}$ particle size) in a transparent plastic cubic container with a size of $4 \mathrm{~cm} \times 5$ $\mathrm{cm} \times 2 \mathrm{~cm}$; where the scattering coefficient is $9.22 \mathrm{~cm}^{-1}$; the anisotropic factor is 0.65 ; and the size parameter $k a$ is 1.98 .

The geometry of a multiple scattering event in a birefringent turbid medium is shown in Fig. 1(b). At each scattering event, the polar angle $\Theta$ and the rotation angle $\phi$ are selected statistically according to the Mie theory [4-6]. We briefly express the Stokes vector of a transmitted photon packet after it has been scattered $n$ times as

$$
\begin{aligned}
\mathbf{S}_{n}^{\mathrm{fs}}\left(x, y ; \mu_{s}, \mu_{a}\right) & =\left[\mu_{s} /\left(\mu_{a}+\mu_{s}\right)\right]^{n} \\
& \times \mathbf{R}\left(\phi_{L}\right) \mathbf{M}\left(\Theta_{n}\right) \mathbf{R}\left(\phi_{n}\right) \ldots \mathbf{M}\left(\Theta_{1}\right) \mathbf{R}\left(\phi_{1}\right) \mathbf{S}_{0} .
\end{aligned}
$$

where $\mu_{s}, \mu_{a}$ are the scattering and absorption coefficients, respectively; $(x, y)$ is the detection point on the lower surface of the turbid medium in the laboratory coordinate; $\mathbf{S}_{0}$ and $\mathbf{S}_{n}^{\text {fs }}$ represent the Stokes vectors of the incident and the transmitted photon, respectively; $\left[\mu_{s} /\left(\mu_{a}+\mu_{s}\right)\right]^{n}$ denotes the remaining energy after the photon has been scattered $n$ times; $\mathbf{R}(\phi)$ is the rotation matrix that connects the two Stokes vectors that describe the same polarization state but with respect to the two reference planes such that one reference plane coincides with the other after a counterclockwise rotation by angle $\phi$ around the direction of light propagation; and $\mathbf{M}(\Theta)$ is the single scattering matrix for each scattering event based on the Mie theory.

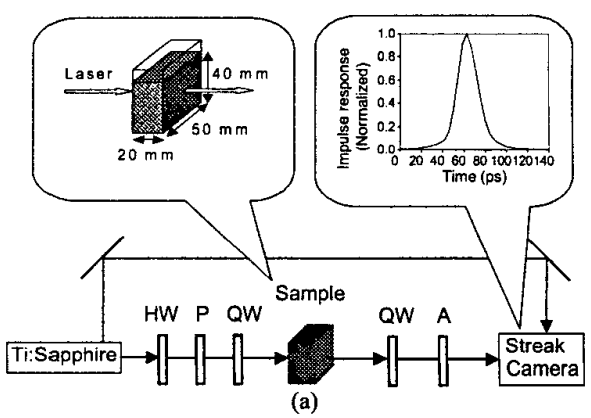




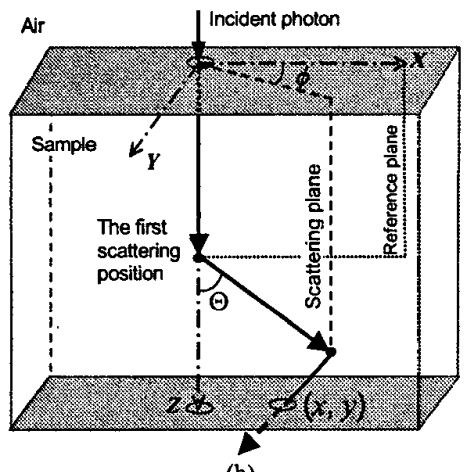

(b)

Fig. 1 (a) The experimental setup. $\mathrm{P}$ polarizer; HW zero order half-wave plate; QW zero-order quarter-wave plate; A analyzer. (b) The geometry of a multiple scattering event in the turbid medium.

\section{RESULTS AND DISCUSSION}

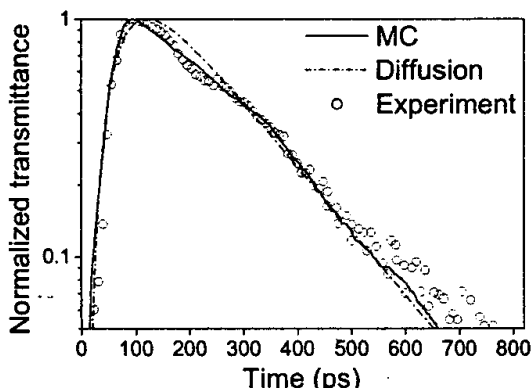

(a)

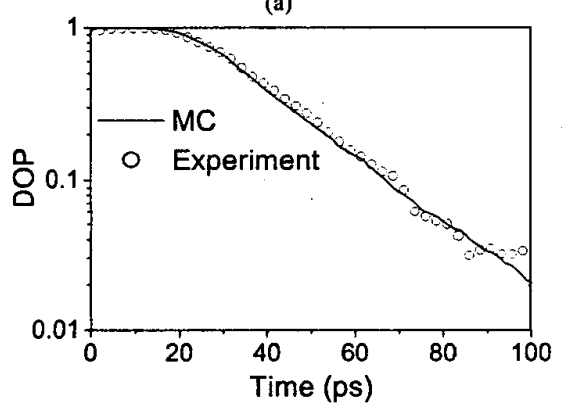

(b)

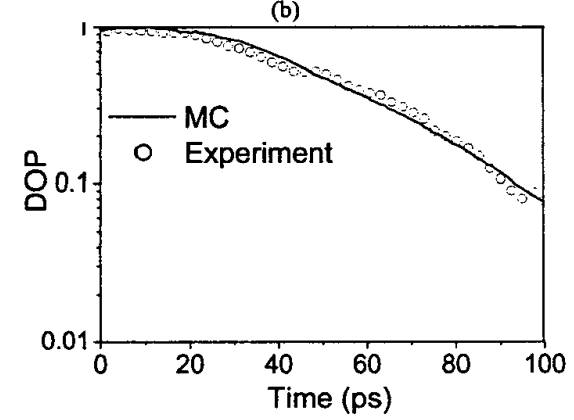

(c)

Fig. 2 (a) The temporal profile of the light transmittance. The timeresolved DOP of transmitted light when the incident light is (b) linearly polarized and (c) circularly polarized, respectively. (Solid curve: MC simulation; scattered symbol: experiment; dashed curves: simulation based on the diffusion theory.)
The temporal profiles of intensity and DOP of transmitted light have been shown in Fig. 2, where the incident light is linearly and circularly polarized respectively for the results in (b) and (c). All the Monte Carlo simulated results match well with the experimental measurements. In Fig. 2(a), the time-resolved transmittance simulated through the diffusion theory matches the experimental result well after $250 \mathrm{ps}$. Photons exiting the sample before that time are lowly scattered photons that have not encountered enough scattering events. Therefore, the distribution of their propagation is not adequately isotropic to be described by the diffusion theory. For both linear and circular incident polarization states, the DOP of transmitted light with respect to the propagation time decays exponentially after about 20 ps. It is obvious that the circularly polarized light preserves its polarization state better than the linearly polarized light during the propagation, which agrees with the analysis of Bicout et. al. [7] for the light propagation in the Mie regime when the size parameter $k a>1.23$.

\section{CONCLUSION}

Compared with the diffusion model, simulations based on the MC technique present a more precise match with the experimental measurements, especially for lowly scattered light component. The satisfying match between the results of simulation and experiment proves the accuracy of this time-resolved MC algorithm. The MC simulations based on the Stokes-Mueller formalism and Mie theory present detailed information of the polarization evolution in the turbid media, which can potentially help to improve the quality of optical imaging that utilizes the ultrafast optics.

\section{ACKNOWLEDGMENT}

This project was sponsored in part by National Institutes of Health grants R01 CA71980 and R21 CA83760, National Science Foundation grant BES9734491, and Texas Higher Education Coordinating Board grant 000512-0123-1999.

\section{REFERENCE}

[1] B. Das, K. Yoo, and R. R. Alfano, "Ultrafast time gated imaging," Opt. Lett, vol. 18, pp.1092-1094, 1993.

[2] J. M. Schmitt, A. H. Gandjbakhche, and R. F. Bonner, "Use of polarized light to discriminate short-path photons in a multiply scattering medium," Appl. Opt., vol. 31, pp.6535-6546, 1992.

[3] C. W. Sun, C. Y. Wang, C. C. Yang, Y. W. Kiang, I. J. Hsu, and C. W. Lin, "Polarization gating in ultrafast-optics imaging of skeletal muscle tissue," Opt. Lett., vol. 26, pp.432-434, 2001.

[4] G. Yao, and L. V. Wang, "Propagation of polarized light in turbid media: simulated animation sequences," Opt. Express, vol. 7, pp.198-203, 2000

[5] X. D. Wang, and L. V. Wang, "Propagation of polarized light in birefringent turbid media: time-resolved simulations," Opt. Express, vol. 9, pp.254-259, 2001.

[6] H. C. van de Hulst, Light Scattering by Small Particles, Dover, New York, 1981.

[ 7 ] D. Bicout, C. Brosseau, A. S. Martinez, and J. M. Schmitt, "Depolarization of multiply scattered waves by spherical diffusers: influence of the size parameter," Phy. Rev., vol. E49, pp.1767-1770, 1994. 